\title{
Measuring Current Profiles in the Textor Tokamak
}

\author{
H. Soltwisch, Jülich
}

(Institut für Plasmaphysik der Kernforschungsanlage Jülich GmbH, Association EURATOM-KFA)

\begin{abstract}
Tokamak devices are presently the most studied objects in controlled thermonuclear fusion research. Their performance depends crucially on the internal magnetic field structure within the high temperature plasma that constitutes the active medium. Only recently have direct measurements of this field structure been achieved routinely.
\end{abstract}

European efforts in controlled nuclear fusion research which are promoted and coordinated by EURATOM, put most emphasis on the tokamak device, which as shown in Fig. 1, is essentially a large transformer with a conducting plasma ring acting as a single-turn secondary winding. The induced current / heats the plasma resistively and generates a poloidal magnetic field $B_{p}$. By combining it with a strong field $B_{t}$ along the current (which is produced by a set of coils surrounding the vacuum chamber), the magnetic field lines become helical and form a set of nested toroidal surfaces onto which the charged plasma particles are confined. A slightly curved vertical field $B_{\mathrm{v}}$ serves to counteract radial expansion forces and to centre the plasma in the vacuum vessel.

Unfortunately, plasmas contained in a tokamak configuration are susceptible to a large number of instabilities. While 'microscopic' disturbances like finegrained turbulences mainly cause an enhanced heat diffusion and thereby deteriorate the confinement of energy, 'macroscopic' instabilities by spontaneous magneto-hydrodynamic deformations of the magnetic field structure can lead to an abrupt termination of the plasma

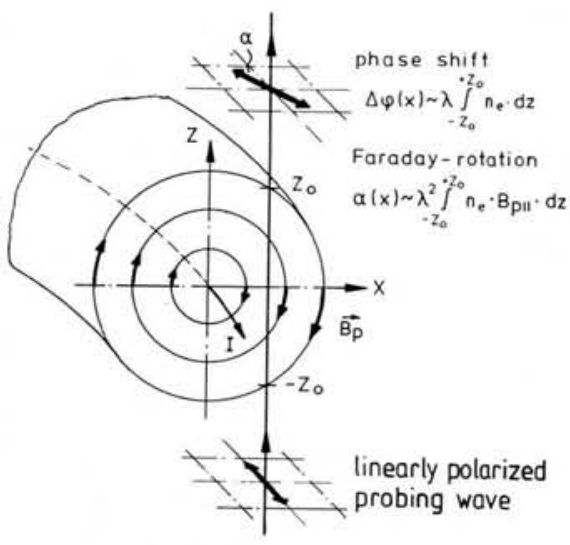

Fig. 2 - Principle of simultaneous density and magnetic field measurements by means of a far-infrared laser beam. current and to a massive strain on the mechanical structure that has to take the suddenly released energy. These latter MHD instabilities limit both the current and particle densities attainable in a given device.

It is thus the current distribution and the associated poloidal field $B$ that play a central role in a tokamak by (i) providing power input to the plasma, (ii) forming a suitable magnetic container, and (iii) determining the appearance of detrimental MHD instabilities. Obviously, a detailed understanding of this key parameter is of prime importance for both the theoretical description and the practical improvement of devices.

Various 'direct' methods have been developed to a 'proof in principle' stage (see Ref. 1), but routine measurements of the poloidal field pattern in a relatively wide range of plasma parameters have been achieved so far only by far-infrared polarimetry. This method is based on the Faraday effect exerted on a linearly polarised electromagnetic wave as it passes through a plasma along a magnetic field line.

As it happens, the waves experience just enough Faraday rotation for sufficiently accurate measurements without being disturbed too much by secondary effects. However, upgrading an existing interferometer for additional polarimetric measurements is a difficult technical task, and reconstructing the local poloidal field from the chord-averaged Faraday rotation data requires extensive calculations. Nevertheless, the feasibility has been demonstrated, and polarimetry is presently regarded as the most appropriate internal field diagnostic on large devices.

\section{Experimental Technique}

Fig. 2 shows schematically a tokamak plasma with a far-infrared laser beam traversing a poloidal cross-section normal to the current I. Upon entry the probing beam is linearly polarised with its

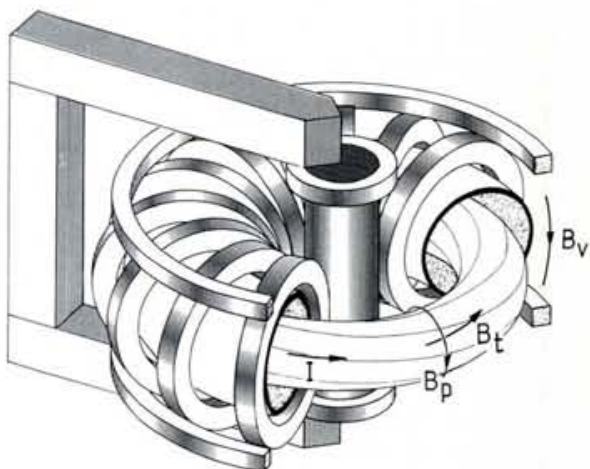

Fig. 1 - Schematic drawing of a tokamak device.

electric field vector parallel to the toroidal magnetic field $B_{t}$. During its passage through the plasma the phase velocity is changed as a function of the local electron density $n_{e}$, and the plane of polarisation is rotated by the magnetic field component $B_{p \mid}$ along the beam path. Thus, emerging from the plasma the beam exhibits a phase shift $\Delta \varphi$ and a Faraday rotation $\alpha$ according to

$\Delta \varphi \sim \int_{-z_{0}}^{+z_{0}} n_{\mathrm{e}} d z, \quad \alpha \sim \int_{-z_{0}}^{+z_{0}} n_{\mathrm{e}} B_{\mathrm{p} \|} d z$.

Both quantities can be measured simultaneously by combining an interferometer with a sensitive polarimeter in a variety of conceivable schemes. Here we shall limit ourselves to a description of the set-up installed on the TEXTOR tokamak.

Let us first consider the interferometric arrangement shown in Fig. 3. It is basically a Mach-Zehnder configuration whose output interference pattern is caused to travel at a constant speed across the detector $D_{1}$ by introducing a slight frequency shift to the reference beam (in Fig. 3 this frequency shift is produced by diffracting the beam off a rotating cylindrical grating). Any changes in the optical path length of the probe beam will modify the travelling speed such that variations of the plasma density will be translated into phase shifts of a convenient low-frequency signal (typically $10 \mathrm{kHz}$ ) delivered by $D_{1}$. Hence, $\Delta \varphi$ is readily obtained from a phase comparator which is fed with the detector output and a reference signal of equal frequency and fixed phase. The latter is generated by splitting off small portions of the reference beam before and behind the rotating grating and mixing them on a second detector $D_{R}$, thereby forming an interferometer without any plasma in its probing arm. In practice, the arrangement provides a resolution of $10^{-2} \times 2 \pi$ at total phase shifts of $\leqslant 5 \times 2 \pi$ occurring in a typical plasma pulse. 


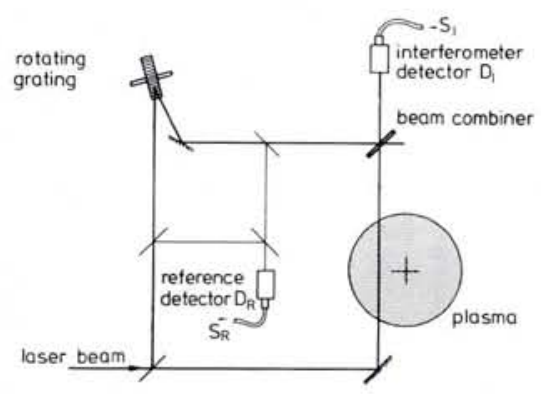

Fig. 3 - Modified Mach-Zehnder interferometer for measuring plasma-induced phase shifts of the probing wave lafter $D$. Véron, Opt. Commun. 10 (1974) 95.

In order to facilitate a simultaneous detection of the Faraday rotation angle, the system has been modified according to Fig. 4. The essential alteration of the basic configuration is the use of a polarising beam combiner which allows us to split off that component of the probing beam that has acquired a polarisation perpendicular to the initial one due to Faraday rotation. By letting it interfere with part of the frequency-shifted reference beam, we obtain another travelling fringe pattern whose contrast depends on the extracted portion of the probe wave. This interference pattern is picked up by the 'polarimeter' detector $D_{p}$ which transforms the modulating intensity into an a.c. signal with an amplitude approximately proportional to the rotation angle $\alpha$. The much larger component of the probing wave that is transmitted by the beam combiner passes on towards the 'interferometer' detector $D$ together with the remaining portion of the reference beam. Since their polarisations are orthogonal, they have to be mixed by an analyser in front of $D_{1}$ in order to produce interference. The halfwave' plate located between the plasma and the beam combiner serves to null the polarimeter signal in the absence of Faraday rotation by adjusting the probing wave polarisation for minimum reflexion at the polariser.

In this set-up, the information on $\Delta \varphi$ is derived from the interferometer signal as described above, whereas an accurate determination of $\alpha$ requires a more elaborate signal processing. In the first place, amplitude variations due to the Faraday effect are rather small and hardly raise the polarimeter signal above the detector noise level. This problem is overcome with the help of modern lockin techniques employing the large and 'clean' interferometer signal to select the right frequency (although at the expense of a reduced time resolution). Besides noise filtering, the lock-in amplifier also helps to determine the sign of the rota- tion angle, since the polarimeter signal undergoes a phase jump of $\pi$ when $\alpha$ passes through zero, whereas the interferometer signal serving as a reference remains unaffected. Next, any fluctuation of the far-infrared beam intensities influences the signal amplitude, which would make a small variation of the laser power indistinguishable from a change of the Faraday rotation angle. These intensity fluctuations have to be taken into account by using the interferometer signal as a power monitor (its amplitude is proportional to $\cos \alpha$ and therefore almost independent of Faraday rotation for typical angles of less than $10^{\circ}$ encountered in these measurements). Finally, the detector responsivities and the conversion factor of the whole signal processing circuit have to be determined by a careful absolute calibration.

In spite of its complexity the polarimetric system is capable of resolving plasma-induced variations of the rotation angle to within $\pm 0.15^{\circ}$ at an integration time constant of only a few milliseconds. Those who are interested in further technical details are referred to Ref. 2.

\section{Data Analysis and Results}

A unique reconstruction of local values of $n_{\mathrm{e}}$ and $B_{\mathrm{p}}$ requires, in principle,
INTERNATIONAL SCHOOL OF PLASMA PHYSICS

"PIERO CALDIROLA"

Varenna 1987

\section{WORKSHOP}

Theory of fusion plasmas

Edited by A. Bondeson, Institute for Electromagnetic Theory, Chalmers University of Technology, Goteborg, E. Sindoni, Dipartimento di Fisica, Università di Milano, Milano, and F. Troyon, CRPPEPFL, Lausanne.

In one volume of about 600 pages, available from February 1988. price US $\$ 80$.

\section{COURSE AND WORKSHOP}

Physics of Mirrors, Reversed Field Pinches and Compact Tori

Edited by S. Ortolani, Istituto Gas Ionizzati del CNR, Padova, and

E. Sindoni, Dipartimento di Fisica, Università di Milano, Milano.

In three volumes of about 1200 pages, available from February 1988,

price US \$ 120 .

Orders should be sent to the Italian Physical Society,

Via L. degli Andalò, 2,

I - 40124 Bologna Bo, Italy.
Fig. 4-Details of the beam recombination as utilized for simultaneous interferometric and polarimetric measurements.

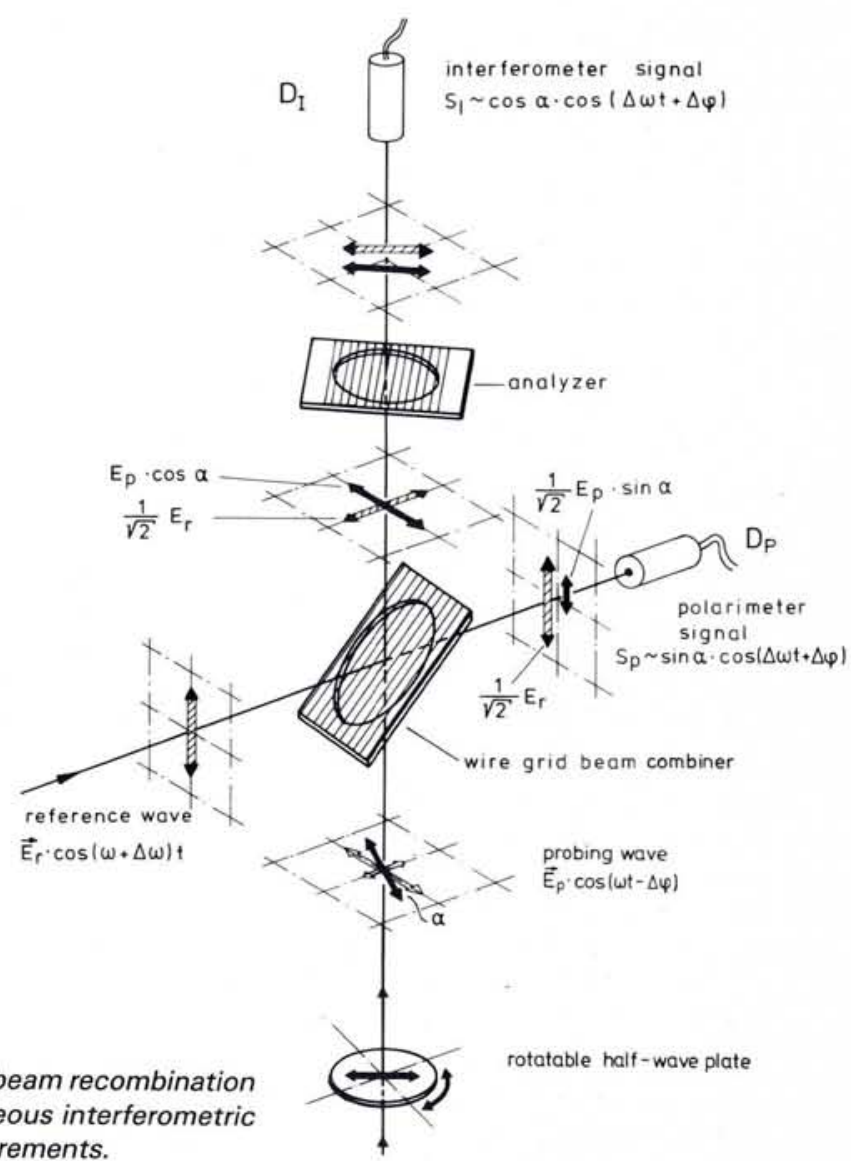


a large number of closely spaced beams traversing the plasma at different angles. In practice, however, a tokamak is only accessible for a few beams preferentially oriented perpendicular to the torus midplane, and the lack of experimental data has to be made up by more or less justified assumptions on the plasma shape and inherent symmetries in the sought-after distributions. To illustrate the basic concept of data analysis, let us consider the simple case of radial symmetry where the local density and poloidal field strength depend only on the distance $r$ from the plasma centre. Here we can divide the plasma crosssection into a number of concentric ring zones on which $n_{\mathrm{e}}$ and $B_{\mathrm{p}}$ are approximately constant. Hence, the path of a probing beam intersecting these zones is split up into small segments with constant plasma parameters, and the integrals, equ. (1), may be expressed as finite sums of these sections. A beam traversing just the outermost zone is represented by two single terms which provide immediately the density and field strength at the plasma edge. This information is then used to calculate the edge contributions to the signals of an adjacent beam passing through the next inner zone, which leaves us after subtraction with the local data in the second ring. Continuing this procedure, we can gradually advance towards the centre.

As for the determination of the poloidal field, we recall that the Faraday effect depends only on the magnetic field component parallel to the beam path rather than the total field. In our cylindrical model this component reduces to $B_{p \|}=(x / r) B_{p}$, where $x$ is the minimum beam distance from the plasma centre, and equ. (1) becomes

$$
\alpha(x) \sim \int_{-z_{0}}^{+z_{0}}(x / r) n_{\mathrm{e}} B_{\mathrm{p}} d z .
$$

Interpreting $(x / r)$ and $n_{\mathrm{e}}$ as weight functions along the chord, we notice that both of them have a maximum at the torus midplane and fall off towards the boundaries, thus emphasizing the information on $B_{p}$ gathered near $z=0$ and attenuating the influence of the outer plasma regions. This feature largely hinders errors from accumulating during the successive evaluation outlined above and permits us to draw fairly accurate information on the plasma centre where the field is rather small.

A more realistic treatment of the experimental data has to account for the toroidal geometry of a tokamak plasma which introduces an outward shift of the plasma centre relative to the boundary and causes the poloidal field strength to
Fig. 5 - Superposition of normalised current density profiles measured in a variety of quasistationary TEXTOR discharges; in reduced co-ordinates the distributions assume roughly a unique shape.

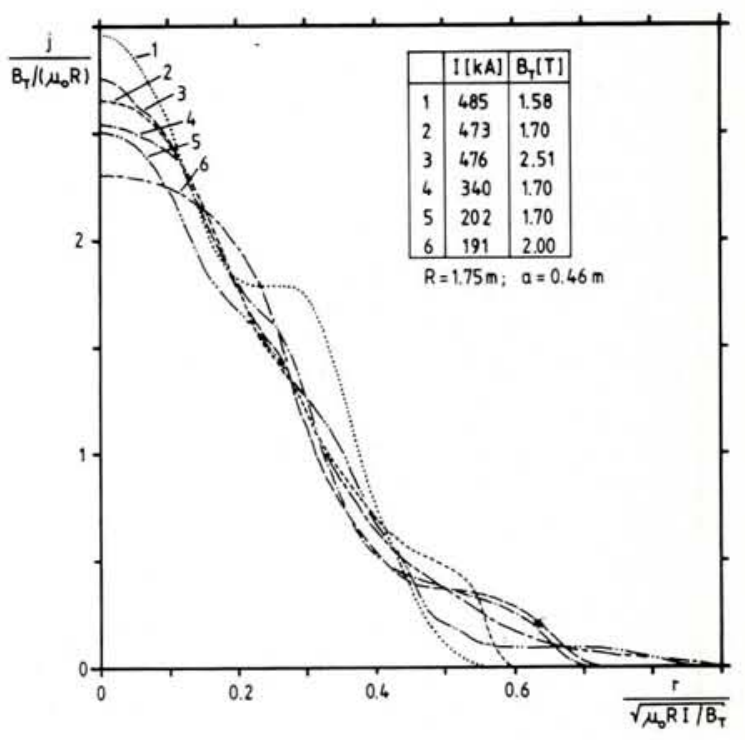

vary on a given magnetic surface which may also be non-circular. Furthermore, secondary effects as for example a slight elliptisation of the probing wave polarisation due to transverse magnetic fields within the plasma have to be considered. These refinements depend, however, to some extent on the poloidal field structure that has to be determined by the analysis procedure. Consequently, appropriate account of these factors can be made only iteratively and requires quite extensive numerical calculations.

Once $B_{p}$ is known, the current profile follows immediately from Ampere's law which relates the field strength on a given magnetic surface to the current flowing through its poloidal cross-section. However, in a tokamak the current density is usually largest in the highly conductive plasma core and decays rapidly towards the boundary. As a consequence, the residual current in the edge region has little influence on $B_{p}$, and we cannot expect to obtain detailed information on the outer part of the current density distribution beyond about $3 / 4$ of the plasma radius. This deficiency, which is common to all techniques aiming at a current profile determination via poloidal field measurement, has prohibited so far conclusive experimental studies of major plasma disruptions.

By contrast, another important instability, which affects only the plasma core and frequently appears as a periodic sawtooth-like modulation of the central electron temperature, is well within the reach of the polarimetric diagnostic. Measurements on the TEXTOR tokamak have challenged the hitherto widely accepted conception of this phenomenon according to which the central part of the current density distribution should be flat inside a well-defined radius with a plateau value depending only on the ex- ternally applied toroidal magnetic field and on the major radius of the plasma torus. In TEXTOR, peaked profiles were found with a maximum value exceeding the prediction by more than $25 \%$. This observation has stimulated refined stability analyses in toroidal geometry which now begin to converge with the experimental findings. Another example to demonstrate the utility of polarimetric measurements concerns the global shape of the current distribution. It has been shown that there exists a unique profile to which a fairly large variety of experimental distributions can be reduced by displaying them in suitably normalised co-ordinates (cf. Fig. 5). Since these normalisation factors depend only on external tokamak parameters, the reduced profile has a universal character independent of a given device for a certain range of plasma conditions whose limits remain to be explored by further investigations.

In summary, the Faraday rotation technique applied on TEXTOR has proved to be a valuable addendum to the set of plasma diagnostic methods commonly employed on modern tokamaks. So far it has provided data on several thousand plasma pulses, and the results have been, for some part, quite unexpected. Thus, there is a good chance that further experimental work in this field, especially with forthcoming polarimetric measurements on the large JET device, will help to resolve some of the remaining problems in tokamak physics.

\section{REFERENCES}

1. Peacock N.J., Diagnostics for Fusion EXperiments, Varenna 1978, ed. E. Sindoni and C. Wharton (Pergamon Press, Oxford) 1979, pp. 367-402.

2. Soltwisch H., Rev. Sci. Instrum. 57 (1986) 1939. 